Christof Heeger, Benjamin Böhm, S.F. Ahmed, Robert Gordon, Isaac Boxx, Wolfgang Meier, Andreas Dreizler, Epaminondas Mastorakos Statistics of Relative and Absolute Velocities of Turbulent Non-premixed Edge Flames Following Spark Ignition

Proc. Combust. Inst. 32 (2009) 2957-2964

The original publication is available at www.elsevier.com

http://dx.doi.org/10.1016/j.proci.2008.07.006 


\title{
Statistics of Relative and Absolute Velocities of Turbulent Non-premixed Edge Flames Following Spark Ignition
}

\author{
C. Kittler ${ }^{1}$, B. Böhm ${ }^{1}$, S.F. Ahmed², R. Gordon ${ }^{1}$, I. Boxx ${ }^{3}$, W. Meier ${ }^{3}$, A. Dreizler ${ }^{1}$, E. \\ Mastorakos $^{2}$
}

1: Mechanical Engineering, TU Darmstadt, Germany

2: Engineering Department, University of Cambridge, UK

3: German Aerospace Center, Stuttgart, Germany

Corresponding author:

S.F. Ahmed

Engineering Department,

University of Cambridge

Trumpington Street

Cambridge CB2 1PZ

UK

Tel: +44 1223766289

FAX: +44 1223332662

Email: sfafsa2@eng.cam.ac.uk 


\begin{abstract}
Turbulent non-premixed edge flame propagation velocities following spark ignition have been measured in the opposed-jet geometry using simultaneous high-speed 2D OH PLIF/PIV. The difference in the thickness of the edge between the flame sheet from the OH PLIF images and the evaporated droplets from the PIV images was constant (about $1 \mathrm{~mm}$ ) and did not have any effects on the calculation of the absolute flame speed, $V_{a b s}$. The local flow velocity in the direction of the flame velocity was calculated and resulted in a measurement of the relative propagation velocity, $V_{r}$. It was found that, after an initial transient due to the plasma expansion, the mean $\left\langle V_{a b s}\right\rangle$ increased with time and radial distance as the flame travelled outwards and $\left\langle V_{a b s}\right\rangle$ increased with the bulk velocity $U_{b}$. The fluctuations in $V_{a b s}$ also increased if $U_{b}$ was high. The mean relative propagation velocity, $\left\langle V_{r}\right\rangle$, for all flames was about $0.75 S_{L}$, but increased slightly to $1 S_{L}$ by the inner burner rim. The rms of $V_{r}$ was close to $\left\langle V_{r}\right\rangle$. The pdf of $V_{r}$ was wide with very little content above $3 S_{L}$ and a non-negligible content at negative relative speeds, i.e. retreating fronts, consistent with 3D-DNS results. Separate analysis of images from successful and failed ignition events shows that the pdf of $V_{r}$ for the failed ignition has relatively lower values than the successful. This pdf peaks at about $0.25 S_{L}$, with higher probability of negative $V_{r}$. The measured value of $\left\langle V_{r}\right\rangle / S_{L}$ is a new result and provides insight on the time taken to fully ignite non-premixed combustion in applications such as the relight of gas turbines.
\end{abstract}

Keywords:

Turbulent non-premixed, edge flame, propagation, counter-flow, ignition. 


\section{Introduction}

In many combustion applications, such as during the relight of an aviation gas turbine, the phenomenon of ignition from a localised spark is important and occurs in a turbulent nonpremixed configuration. Flame propagation plays a crucial role in the success or failure of ignition. In laminar flows, the flame propagates with a triple flame front, but under high strain, the two branches of the propagating triple flame front may collapse into a more compact configuration called an edge flame [1-4]. These edge flames are reactive-diffusive waves that propagate probably along the stoichiometric mixture fraction contour. Their speed depends on various parameters, such as the Lewis number, mixture fraction gradient [5], heat release rate [6,7] and flow redirection [7,8]. Previous experimental studies, all of them in 2D, have attempted to measure the propagation speed in laminar igniting non-premixed edge flames, for example in the opposed-jet flow geometry using simultaneous single-shot $\mathrm{CO} / \mathrm{OH}$ PLIF and PIV $[9,10]$ and in laminar jet flames using simultaneous single-shot OH-PLIF and Rayleigh scattering [8]. The relative propagation speed was found to be close to the laminar burning velocity of a stoichiometric unstrained premixed flame, $S_{L}$, or slightly less. No evidence of a negative propagation velocity was found in edge flames associated with extinction 'holes' in a laminar counterflow diffusion flame [9].

In turbulent non-premixed flames, the propagation speed of the edge flame is likely to be affected by the turbulence. The statistics of edge flames in turbulent non-premixed flows have been little studied. Simultaneous single-shot 2-D CH/OH PLIF and PIV was used to investigate the edge flame propagation in turbulent lifted jets [11]. Measurements from single-shot OH-PLIF images of spark-ignited methane edge flames in a turbulent counterflow allowed a crude estimation of the mean propagation speed relative to the flow ahead of the edge to be about $0.3 \mathrm{~m} / \mathrm{s}$, also slightly less than the $S_{L}$ of methane $(0.4 \mathrm{~m} / \mathrm{s})$ [12]. Similar results have been found by a 3-D DNS study of edge flame propagation in turbulent 
inhomogeneous igniting mixing layers [13], where a large scatter in the local value of the relative edge flame speed has been observed. This scatter has been attributed to the local fluctuations of scalar dissipation, curvature and fuel mass fraction gradients. These simulations and others (e.g. [7]) quantified the 'edge displacement speed', which was defined as the speed at which the fuel mass fraction iso-surface moves with respect to an initially coincident material surface at the triple point. This quantity can be approximated by the magnitude of the absolute (i.e. relative to a fixed framework) velocity of the edge flame minus the component of the flow velocity in the direction of the edge flame velocity (the approximation arises due to the inclination of the normal to the flame front relative to the gradient of the mixture fraction at the triple point [13]). Both these speeds can be measured with a simultaneous timeresolved sequence of the flame front position and the fluid velocity at the same location. In lifted jet flames, 2-D cinema-PIV measurements at framing rates up to $8000 \mathrm{fps}$ were used to measure the absolute and relative speed of the flame base (the relative speed was called by these authors 'propagation speed') and it was found that the mean propagation speed was not significantly affected by the turbulence intensity [14], but a large scatter was evident between individual measurements (albeit not quantified in detail). Note that both fluid and flame edge velocities were taken in the axial (jet) direction only, rather than resolved in the direction normal to the flame edge, as was done in laminar triple [8] and edge flames [15] and in the DNS [13], possibly due to the difficulty in identifying the edge flame normal when the triple flame becomes a thin edge flame in the turbulent flow. In the absence of a 3-D cinema-PIV and of a simultaneous mixture fraction measurement, a direct measurement of the edge displacement speed is not feasible, but a 2-D measurement can result in an approximate estimation of the relative and absolute edge flame speed that can sufficiently highlight the effects of turbulence on these quantities.

The counter-flow configuration is ideal to examine turbulent non-premixed edge flames due to its compact domain and simple flow structure. Counterflow flames have been 
extensively studied in terms of extinction, flow field and scalar distributions [16,17]. Based on the recent improvements in solid-state diode-pumped lasers, PIV and PLIF diagnostics are adapted and extended to operate at high repetition rates (in the $\mathrm{kHz}$ regime). The present work uses such diagnostics to provide simultaneous measurements of the speed of a turbulent nonpremixed edge flame front and the local flow velocity ahead of the edge flame, which allows an instantaneous measurement of the relative propagation speed, following localised ignition at the centreline of the burner. In contrast to the lifted jet flames, here the mean radial flow and the travelling edge flame move in the same direction. The stochastic nature of ignition of this flame has been presented previously [12] and here, comparison of the relative velocity associated with failed and successful ignition events will also be presented. The results allow insights into whether advection of the flame by the flow or flame propagation relative to flow is mostly responsible for the overall ignition of turbulent non-premixed flames.

\section{Experimental Methods}

\section{$\underline{2.1 \text { Apparatus }}$}

The work has been performed on an opposed-jet burner (Fig.1), fully described in Ref. [17], installed at the Technical University of Darmstadt. The burner comprises two straight nozzles of inner diameter $D=30 \mathrm{~mm}$, surrounded by co-flows of nitrogen of diameter $D_{0}=60$ mm and velocity $0.3 U_{b}$, where $U_{b}$ is the bulk velocity of the upper jet. The nozzles are separated by a distance $H=D$. The upper nozzle carries air, while the other carries methaneair mixtures. The degree of premixing in the fuel nozzle is described by the equivalence ratio, $\Phi$, which is 2.0 and 3.18 for the flames studied here. Despite this high premixedness, the flame has a non-premixed character because additional air from the top stream is needed for combustion. Table 1 shows the flow conditions investigated. Perforated plates with $45 \%$ 
solidity and hole size of $4 \mathrm{~mm}$ were placed $50 \mathrm{~mm}$ upstream of the nozzle exits to promote turbulence. To achieve a symmetric flow, the momentum flow rates of the two jets were kept equal. The integral lengthscale, $L_{t}$, at the exit of the nozzles was about $4.7 \mathrm{~mm}$ based on hot wire measurements. Previous velocity measurements show that the turbulence intensity at the exit of the nozzles, $u^{\prime} / U_{b}$, is about $10 \%$. At the stagnation region, $u^{\prime} / U_{b}$ increases to about $40 \%$ and the radial fluctuations $v^{\prime} / U_{b}$ to about $20 \%$ [18].

An inductive ignition system was used to produce repeatable sparks whose energy and duration could be varied independently. The main characteristics of the unit can be found in [19]. The spark was created between two tungsten electrodes of $1 \mathrm{~mm}$ diameter with pointed edges to reduce the heat loss and placed in a way to ensure minimum disturbance to the flow [12]. The spark duration, energy and gap were $500 \mu \mathrm{s}, 200 \mathrm{~mJ}$ and $2 \mathrm{~mm}$, respectively. This spark was used to ignite the flames in two locations: at $r=0, z=0$ (the stagnation point) and at $r=4, z=0$, where $r, z$ are the radial and axial coordinates, respectively,

\section{$\underline{2.2 \text { Measurement techniques }}$}

Various laser techniques were used. The Mie-scattering from aerosol droplets with a diameter of about $1 \mu \mathrm{m}$ and seeded through both nozzles was used for high-speed PIV measurements. For these experiments, two independently-controlled diode-pumped frequency-doubled $\mathrm{Nd}: \mathrm{YVO}_{4}$ slab lasers (EdgeWave, IS4II-DE) in one housing, that were flexible in generating double-pulses, were used. The Q-switched edge-pumped slab design is capable of yielding $21.3 \mathrm{~W}$ each at repetition rates up to $32.5 \mathrm{kHz}$ and $8.5 \mathrm{~ns}$ pulse duration, but was limited to $10 \mathrm{kHz}$ (3 W energy) for the present experiments. For the simultaneously running PLIF/PIV lasers, the repetition rate was $2.5 \mathrm{kHz}$.

For OH-PLIF experiments, a frequency doubled diode-pumped Nd:YLF slab laser (EdgeWave, IS 8II-E, $523 \mathrm{~nm}$ ) was operated at $2.5 \mathrm{kHz}$ to pump a tuneable dye laser. The Q- 
switched edge-pumped slab design yielded up to $17.5 \mathrm{~W}$. This corresponded to single pulse energy of $3.4 \mathrm{~mJ}$ and the pulse duration was $8.8 \mathrm{~ns}$. The dye laser was based on a conventional system designed originally for $10 \mathrm{~Hz}$ operation (Radiant Dyes, NarrowScan). To adapt the dye laser to the needs of high repetition rates, several modifications were essential, (see [20] for details). The maximum output power achieved at $2.5 \mathrm{kHz}$ was $120 \mathrm{~mW}$, yielding a pulse energy of $48 \mu \mathrm{J}$. The dye laser was tuned to $\mathrm{Q}_{1} 6$ line of $\mathrm{OH}$ within the $\mathrm{A}^{2} \Sigma-\mathrm{X}^{2} \Pi(1-0)$ band. The laser sheet was approximately $15 \mathrm{~mm}$ high and $0.2 \mathrm{~mm}$ thick for the OH-PLIF, while it was $20 \mathrm{~mm}$ high and $1 \mathrm{~mm}$ thick for the PIV.

Signals from simultaneously recorded Mie-scattering and fluorescence were monitored by two CMOS cameras (LaVision, HSS5) from the same side of the burner. The PIV camera was placed at $13^{\circ}$ off-axis, while the OH-PLIF camera was perpendicular to the laser sheet (Fig. 1). A Scheimpflug adaptor (an optical device) was used to compensate for the PIV camera angle. The active array area for both cameras was $1024 \times 1024$ pixels. The image domain was $25 \times 25 \mathrm{~mm}$ starting from the centreline. The A/D conversion had an effective dynamic range of 9 bit. The PLIF camera had a lens-coupled two stage image intensifier LaVision High-Speed IRO (Intensified Relay Optics). The PLIF signal was collected by a UV lens (Bernhard Halle Nachfl GmbH) with a focal length of $100 \mathrm{~mm}$ fitted with a Scott glass UG11 filter. Both laser systems and the spark were triggered simultaneously.

About 200 high-speed PIV movies were recorded for different ignition events with the spark at $(r, z)=(0,0)$, starting from the spark's end until the flame was fully spread across the burner. About 100 simultaneous high-speed PIV/OH-PLIF movies were recorded for flame TOJ1 to quantify the spatial distance between the flame front inferred from the OH-PLIF movies and the evaporated droplet contours from the PIV movies. In this case, the spark was at $r=4, z=0$, but the image domain for both cameras was limited to avoid any damage to the PLIF intensifier by the spark’s intense luminosity. 
The 2-D absolute flame front velocity was calculated using a frame-by-frame line correlation technique. A contour was identified at the boundary of the evaporated region for each frame. Evenly-spaced, overlapping windows were defined and centered along this contour. The cross-correlation of each contour subsection and the contour subsection from the next frame that fell within the defined window was calculated. To obtain the normal direction of the velocity vector, a 90-degree rotation of the correlation was auto-correlated with itself. The sum of this result and the original correlation yielded a distinct peak. The distance from center of the window to this peak is equivalent to the average positional shift of the flame front.

Uncertainty in the velocity measurements can be introduced from the contour identification algorithm (which is linked to the oil droplet resolution and spacing), the line correlation algorithm, and the identification of the location of the maximum absolute velocity. Analysis of the propagation of laminar edge flames with a strain of $175 \mathrm{~s}^{-1}$ provides a conservative estimate of the precision of about $0.03 \mathrm{~m} / \mathrm{s}$ for absolute velocity magnitudes between 0.3 and $3.1 \mathrm{~m} / \mathrm{s}$. The fluid velocity measurement precision is estimated at about 0.02 $\mathrm{m} / \mathrm{s}$.

\section{Results and Discussion}

\subsection{Edge flame propagation}

Following the spark, a spherical kernel was created that reached a diameter of about 3 mm when the spark ended. The kernel then formed the flame edge that spread radially, probably along the stoichiometric mixture fraction $\left(\xi_{\mathrm{st}}\right)$ iso-line, as a thin sheet with about 1.5 mm thickness until it filled the whole stagnation plane between the two nozzles. Figure 2 shows simultaneous OH PLIF/PIV images of one successful ignition event. It can be observed 
that there is a difference between the thickness of the flame sheet visualised in the OH-PLIF images and the thickness of the evaporated droplet region surrounding the flame in the PIV images. This difference was between 0.5 and $1 \mathrm{~mm}$ ahead of the flame edge and is due to the droplet evaporation in the preheat zone of the flame, as also observed in [14]. The propagating edge fluctuated following the axial turbulent fluctuations and the flapping of the instantaneous $\xi_{\text {st. }}$ In case of failed ignition events, either no flame kernel was created after the spark or an edge flame propagated for about 2 to $3 \mathrm{~ms}$ and then the whole flame was quenched.

Using PIV images like those in Fig. 2, the leading edge of the propagating flame was defined by the point that had the maximum absolute velocity ( $V_{a b s}$ in Fig. 2), as described earlier, such as point $A$ in the first image in Fig. 2. The difference in the flame thickness between the OH-PLIF and the PIV image did not have any effect on the location of point $A$. This was checked by tracking the location of point $A$ on the PIV images back with time and it was found that it resulted in the same location on the OH-PLIF image. The difference in $V_{a b s}$ between these two locations was negligible. In addition, this difference was constant throughout the whole propagation event, which gave confidence to the use of the evaporated droplet contour of the PIV image to calculate the edge flame speed. Once the magnitude and the direction of $V_{a b s}$ on point $A$ were determined, the magnitude and the direction of the fluid velocity vector $V_{f}$ was quantified at point $A$. Then, to get the relative propagation velocity $V_{r}$, the fluid velocity $V_{f}$ was projected in the direction of $V_{a b s}$ following the method used by Ref. [8], so $V_{r}=V_{a b s}-V_{f} \cos \theta$, where $\theta$ is the angle between $V_{f}$ and $V_{a b s}$.

\section{$\underline{3.2 \text { Absolute and relative propagation speeds }}$}

The absolute (i.e. relative to the laboratory coordinates) and the relative (to the flow) propagation velocities of the edge flame have been measured and calculated following the 
method described previously. In Fig. 3, a few individual traces of absolute and relative flame edge speeds are shown, together with their mean, as typical examples of the evolution of these quantities with time. It is evident that the absolute velocity increases with time, that individual realisations show an edge velocity that fluctuates, that the relative velocity is smaller than the absolute with a mean that is relatively constant with time, and that the instantaneous relative velocity of the edge as it expands also contains significant temporal fluctuations. A statistical approach is therefore necessary.

Figure 4 shows the mean $\left\langle V_{a b s}\right\rangle$ and rms of the absolute propagation velocity, $V_{a b s, r m s,}$ as a function of the time and radial distance from the spark. The averaging is performed only using movies with ignition events resulting in a wholly ignited flame. The $\left\langle V_{a b s}\right\rangle$ for flames TOJ1 and TOJ2 are almost identical, i.e. regardless of the difference in $\Phi$. Altering the premixedness changes the location of the $\xi_{\text {st }}$ iso-line, but does not seem to change the edge flame speed. $\left\langle V_{a b s}\right\rangle$ for both flames is initially high (about $3 \mathrm{~m} / \mathrm{s}$ ) probably due to the expansion induced by the spark plasma. Then, $\left\langle V_{a b s}\right\rangle$ decreases quickly, followed by a gradual increase of $\left\langle V_{a b s}\right\rangle$, as the flame expands and is now found in increasingly larger radius where the radial flow velocity increases, Fig. 4a. $\left\langle V_{a b s}\right\rangle$ reaches about $2.7 \mathrm{~m} / \mathrm{s}$ after $7 \mathrm{~ms}$ from the spark. For flame TOJ3, after the initial rapid decay from about 3.3 to $1.75 \mathrm{~m} / \mathrm{s},\left\langle V_{a b s}\right\rangle$ increases more rapidly than in TOJ1 and TOJ2 and the whole propagation across the nozzle finishes in just 5 ms. This is due to the higher $U_{b}$ in TOJ3, which shows that the absolute propagation speed is mainly driven by the radial velocity.

A similar behaviour can be observed when $\left\langle V_{a b s}\right\rangle$ is plotted versus the radial location of the edge, Fig. 4b. The initial effect of the spark finishes at about $3.6 \mathrm{~mm}$ from the spark position. At this location, the edge flame speed starts to increase gradually until it reaches about $3.3 \mathrm{~m} / \mathrm{s}$ for TOJ1 and TOJ2, and about $3.8 \mathrm{~m} / \mathrm{s}$ for TOJ3, where the flame passes the 
inner radius of the burner $(D / 2=15 \mathrm{~mm})$ and starts to be affected by the nitrogen co-flow. Again, the rate of increase in $\left\langle V_{a b s}\right\rangle$ with distance is highest for the TOJ3 flame.

The rms of $V_{a b s}$ is included in Fig. 4. The high extensional strain and radial turbulence are mostly responsible for the fluctuations in the edge flame position and, as a result, in $V_{a b s, r m s}$. This effect can be observed in Fig. $4 \mathrm{a}$ as the rms grows with time. The rate of increase in the rms of flame TOJ2 is slightly higher than that of TOJ1 even though they have the same $U_{b}$. The rms of flame TOJ3 is higher than those of the other two flames due to the higher $U_{b}$. When plotted against radial position (Fig. 4b), the rms of the three flames appear closer to each other, but again, the rms of TOJ3 and TOJ2 are slightly higher than that of TOJ1.

The mean $\left(\left\langle V_{r}\right\rangle\right)$ and rms $\left(V_{r, r m s}\right)$ of the relative propagation velocity $V_{r}$ are shown in Fig. 5. The initial effect of the spark discharge on $V_{r}$ is also evident. The $\left\langle V_{r}\right\rangle$ curves for the three flames almost collapse to a single curve. The mean relative velocity decreases from about $1.8 \mathrm{~m} / \mathrm{s}$ to about $0.3 \mathrm{~m} / \mathrm{s}$ in about $1 \mathrm{~ms}$ after the spark. This value is slightly lower than the stoichiometric laminar burning velocity $S_{L}$ for methane. Following this period, $\left\langle V_{r}\right\rangle$ stays relatively constant until about $4 \mathrm{~ms}$. Then, it starts to increase slightly to reach about $0.4 \mathrm{~m} / \mathrm{s}$ $\left(\approx S_{L}\right)$ by the end of the propagation across the burner.

These results are in good agreement with previous estimates of the mean edge propagation speed in turbulent counter-flow flames [12] and with the measurements in the base of turbulent lifted jet flames [14] (based on 2-D images) and with the 3-D DNS data for turbulent inhomogeneous mixing layers [13]. It also agrees with the suggestion that using the corrected local flow velocity in the calculation of $V_{r}$ results in $V_{r}$ to be independent from flow velocity [8]. The mean $\left\langle V_{r}\right\rangle$ in the present turbulent flows is also close to the laminar flame values measured in jets [8] and opposed-jet flows [3,7,9,15].

The rms of $V_{r}$ are also included in Fig. 5. With time from the spark initiation, the rms values for all flames do not seem to be strongly affected by the spark discharge at the 
beginning, consistent with the view that initially a flame kernel behaves as a laminar flame. The rms of $V_{r}$ then stays relatively uniform until $4 \mathrm{~ms}$ for TOJ1 and TOJ2, and $2.5 \mathrm{~ms}$ for TOJ3 and are close to the mean values. Then, the rms increases towards the end of the propagation, which happens at about $7 \mathrm{~ms}$ for flames TOJ1 and TOJ2 and at $5 \mathrm{~ms}$ for TOJ3, probably due to the turbulent mixing with the nitrogen co-flow. Similar behaviour can be observed for the rms trend as a function of $r$ (not shown here).

Figure 6 gives more detail on the statistics of $V_{r}$ by showing the pdf's of $V_{r}$. The pdf shifts towards smaller $V_{r}$ after the initial spark effects have died out. The pdf then relaxes to a shape that seems relatively independent of time or flow condition. The relative flame speed has a large scatter, but does not often exceed $3 S_{L}$. There is non-negligible content at negative relative speeds, i.e. retreating fronts, and this content increases with $U_{b}$. Increasing the bulk velocity also causes an increase in the content at the positive tail of the pdf.

Despite being taken from 2-D images, the present findings are fully consistent with 3D DNS [13] and show that turbulent edge flames have a local propagation speed (relative to the flow) with large variations. These variations seem to increase with the magnitude of the turbulent fluctuations. However, their mean value is around $0.75 S_{L}$ with only a mild dependence on bulk velocity. The absolute propagation speed has a large scatter about the mean, which explains the large scatter in flame position at the same time from the spark in individual spark events [12]. The mean absolute speed is much higher than the relative, which suggests that in this flow the mean radial convection is mostly responsible for overall flame establishment. The small magnitude of the relative speed also explains the long time necessary to ignite fully a turbulent jet non-premixed flame [19] and is consistent with the very small ignition probability observed in the ignition of non-premixed recirculating flows if the spark is placed in locations where the mean convection hinders flame spreading to the base of the recirculation zone [21]. 


\section{$\underline{3.3 \text { Failed and successful ignitions }}$}

The data shown previously were compiled from PIV movies of successful ignitions. To identify the reasons for failed propagation events, which contribute to the failed ignition events (not having a kernel at all following the spark is the other reason for failure), movies from failed ignition were also analysed. Figure 7 shows the pdf of $V_{r}$ separately for only failed and only successful events. $V_{r}$ was quantified over the whole propagation time until the flame quenched (failed events) or until the flame passed the image domain (successful events). It can be observed that the failed ignition shows a peak in the pdf at low $V_{r}$, about $0.1 \mathrm{~m} / \mathrm{s}$, with a long tail in the negative $V_{r}$ range. Successful ignitions have a pdf that peaks at a higher $V_{r}$ $(0.3 \mathrm{~m} / \mathrm{s})$ with negative values having a smaller probability. Failed ignitions are therefore associated with an increased likelihood of retreating fronts.

The pdfs of the strain rate at the flame edge and of the vorticity ahead of the flame (not shown) do not reveal any major differences between failed and successful ignitions. Hence the reason for the lack of propagation for the failed events is not clear from the present data. DNS suggests [13] that in a turbulent flow the mixture fraction gradient and the flame's curvature mostly determine the instantaneous edge displacement speed and hence simultaneous mixture fraction measurements that can give the scalar dissipation might help in explaining the failed ignition events.

\section{Conclusions}

Absolute and relative propagation velocities of turbulent non-premixed edge flames propagating across a turbulent non-premixed counterflow burner have been measured with a high-speed OH PLIF/PIV system. The main conclusions are: first, after an initial period of 1 ms that is affected by the spark, $\left\langle V_{a b s}\right\rangle$ increased from 3 to $7 S_{L}$ until the flame reached the 
radius of the nozzle. Second, $\left\langle V_{a b s}\right\rangle$ was not affected by changing the degree of premixedness of the flow, but it was increased by an increase in $U_{b}$. This shows that $\left\langle V_{a b s}\right\rangle$ is mainly driven by the radial velocity rather than by propagation. Third, the rms of $V_{a b s}$ was about $30 \%$ of the mean value; with a slight increase at high $U_{b}$ due to the increase in the turbulent velocity fluctuations. Fourth, the mean relative velocity $\left\langle V_{r}\right\rangle$ for all flames was about $0.75 S_{L}$, but increased slightly to $1 S_{L}$ away from the burner centreline. The rms of $V_{r}$ was close to $\left\langle V_{r}\right\rangle$. The pdf of $V_{r}$ is wide. $V_{r}$ does not often exceed $3 S_{L}$ and the pdf has a non-negligible content at negative relative speeds, i.e. retreating fronts, which is fully consistent with DNS results. Finally, The failed ignition is associated with a pdf that peaks at $V_{r} \approx 0.25 S_{L}$, with an increased probability of negative values.

\section{Acknowledgements}

Financial support by Deutsche Forschungsgemein-schaft (DFG) through Sonderforschungsbereich 568, projects A4 and B1, Graduiertenkolleg 1344/1 and project DR 374/5-1 is kindly acknowledged. The team at Cambridge acknowledges the EU project TIMECOP-AE (AST5-CT-2006-030828) and thanks Dr. N. Chakraborty of Liverpool University for many insightful discussions on edge flames. 


\section{References}

1. J. Buckmaster, Prog. Energ. Combust. Sci. 28 (2002) 435-475.

2. V. Favier, L. Vervisch, Combust. Flame 125 (2001) 788-803.

3. V.S. Santoro, A. Liñán, A. Gomez, Proc. Combust. Inst. 28 (2000) 2039-2046.

4. T.G. Vedarajan, J. Buckmaster, Combust. Flame 114 (1998) 267-273.

5. L.J Hartley, J.W. Dold, Combust. Sci. Technol. 80 (1991) 23-46

6. G.R. Reutsch, L. Vervisch, A. Liñán, Phys. Fluids 7 (1995) 1447.

7. T. Echekki, J.H. Chen, Combust. Flame 114 (1998) 231-245.

8. M.K. Kim, S.H. Won, S.H. Chung, Proc. Combust. Inst. 31 (2007) 901-908.

9. G. Amantini, J.H. Frank, A. Gomez, Proc. Combust. Inst. 30 (2005) 313-321.

10. W.F. Carnell Jr, M.W. Renfro, Combust. Flame 141 (2005) 350-359.

11. K.M. Lyons, Prog. Energ. Combust. Sci. 33 (2007) 211-231.

12. S.F. Ahmed, R. Balachandran, E. Mastorakos, Proc. Combust. Inst. 31 (2007) 1507-1513.

13. N. Chakraborty, E. Mastorakos, Phys. Fluids 18 (2006) 105103.

14. A. Upatnieks, J.F. Driscoll, C.C. Rasmussen, S.L. Ceccio, Combust. Flame 138 (2004) 259-272.

15. C.E. Frouzakis, A.G. Tomboulides, J. Lee, K. Boulouchos, Combust. Flame 130 (2002) 171-184.

16. E. Mastorakos, A. M. K. P. Taylor, J. H. Whitelaw, Combust. Flame 91 (1992) 40-54.

17. D. Geyer, A. Kempf, A. Dreizler, J. Janicka, Combust. Flame 143 (2005) 524-548.

18. R.P. Lindstedt, D.S. Luff, J.H. Whitelaw, Flow Turb. Combust.74 (2005) 169-194.

19. S.F. Ahmed, E. Mastorakos, Combust. Flame 146 (2006) 215-231.

20. C. Kittler, A. Dreizler, Appl. Phys. B 89 (2007) 163-166.

21. S.F. Ahmed, R. Balachandran, T. Marchione, E. Mastorakos, Combust. Flame 151 (2007) 366-385. 


\section{List of Figure Captions:}

Fig. 1: Test rig and the optical layout for simultaneous high-speed OH PLIF/PIV imaging.

Fig. 2: Simultaneous OH-PLIF (left) and PIV (right) images at different time delays after the spark from one ignition event recorded at $2.5 \mathrm{kHz}$. Spark at $r=4, z=0$. Image domain is $25 \times 8$ $\mathrm{mm}$. The axis is located on the right end of the PIV images and the fuel comes from below.

Fig. 3: Instantaneous and mean absolute speed and relative of edge flames against time from the spark initiation for TOJ1. Spark at $r=0, z=0$.

Fig. 4: Mean and r.m.s. of the absolute speed of the edge flames against (a) time from the spark initiation and (b) distance from the spark location. Spark at $r=0, z=0$.

Fig. 5: Mean and r.m.s. of the speed of the edge flames relative to the flow against time from the spark initiation. Spark at $r=0, z=0$.

Fig. 6: Probability density function (PDF) of the relative propagation velocity of (a) the three flames at $4 \mathrm{~ms}$ after the spark, and (b) flame TOJ1 at different time delays from the spark.

Fig. 7: Comparison between the pdf of the relative velocity for the successful and failed ignition events for flame TOJ1. Spark at $r=0, z=0$ 


\section{TABLES}

\section{Table 1}

Flow conditions of the investigated flames.

\begin{tabular}{|l|l|l|l|}
\hline Flames & TOJ1 & TOJ2 & TOJ3 \\
\hline Re & 5000 & 5000 & 6000 \\
\hline Equivalence ratio $\Phi$ & 2.0 & 3.18 & 2.0 \\
\hline Bulk velocity $U_{b}(\mathrm{~m} / \mathrm{s})$ & 2.55 & 2.55 & 3.1 \\
\hline Bulk strain rate $\left(\mathrm{s}^{-1}\right)$ & 175 & 175 & 211 \\
\hline
\end{tabular}

Single-column width 


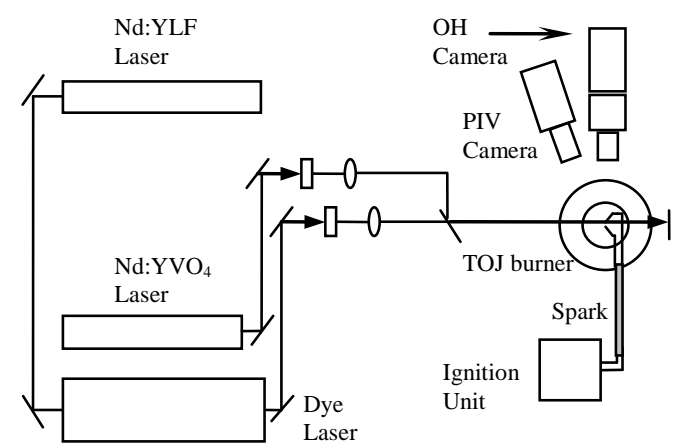

Fig. 1: Test rig and the optical layout for simultaneous high-speed OH PLIF/PIV imaging.

Single-column width 


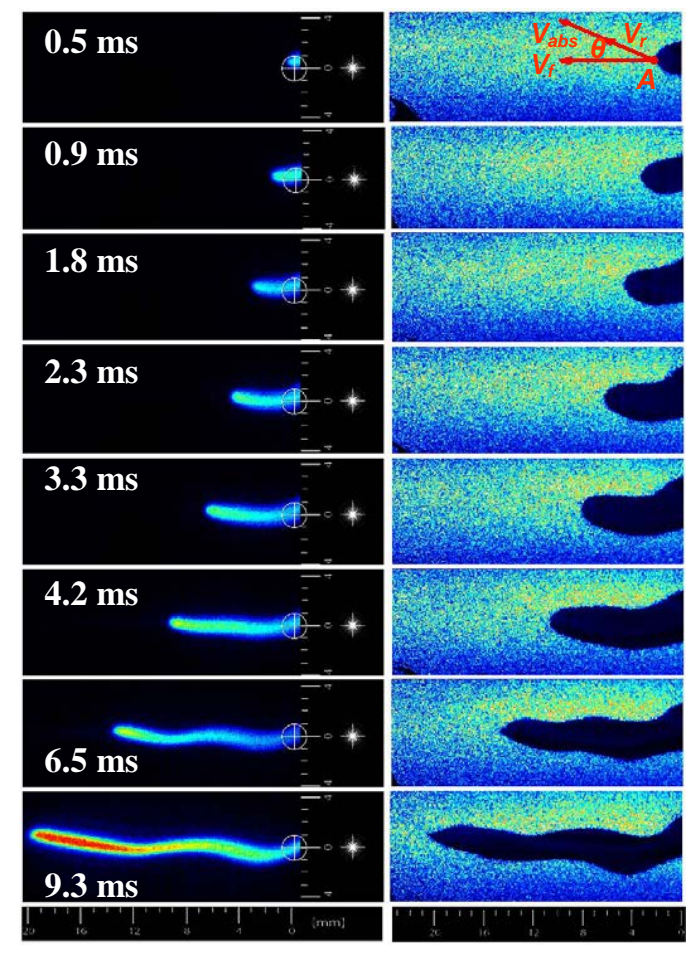

Fig. 2: Simultaneous OH-PLIF (left) and PIV (right) images at different time delays after the spark from one ignition event recorded at $2.5 \mathrm{kHz}$. Spark at $r=4, z=0$. Image domain is $25 \times 8$ $\mathrm{mm}$. The axis is located on the right end of the PIV images and the fuel comes from below.

Single-column width

Colour figure in electronic versions only 


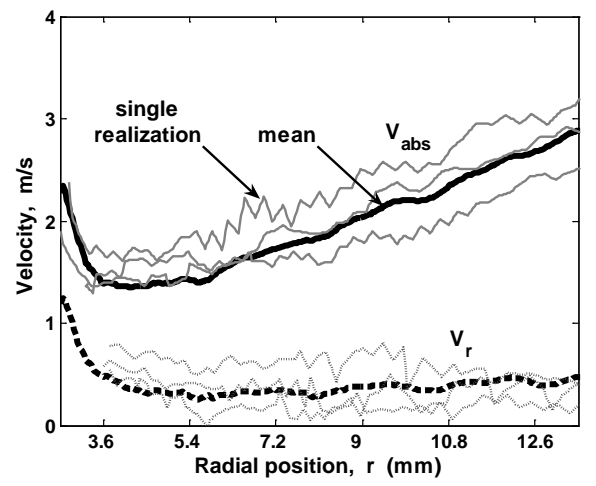

Fig. 3: Instantaneous and mean absolute speed and relative of edge flames against time from the spark initiation for TOJ1. Spark at $r=0, z=0$.

Single-column width 


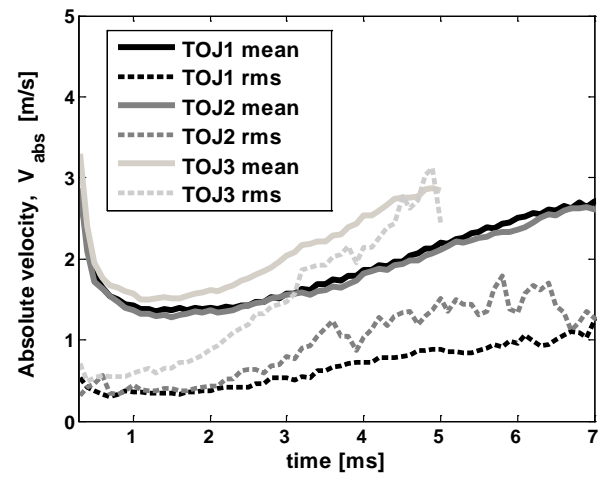

(a)

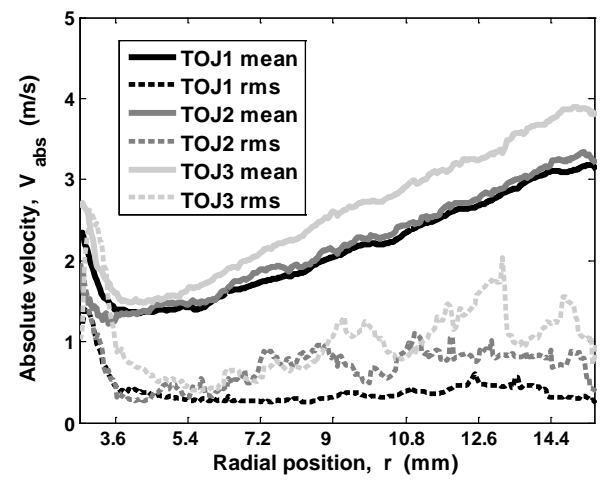

(b)

Fig. 4: Mean and r.m.s. of the absolute speed of the edge flames against (a) time from the spark initiation and (b) distance from the spark location. Spark at $r=0, z=0$.

Single-column width 


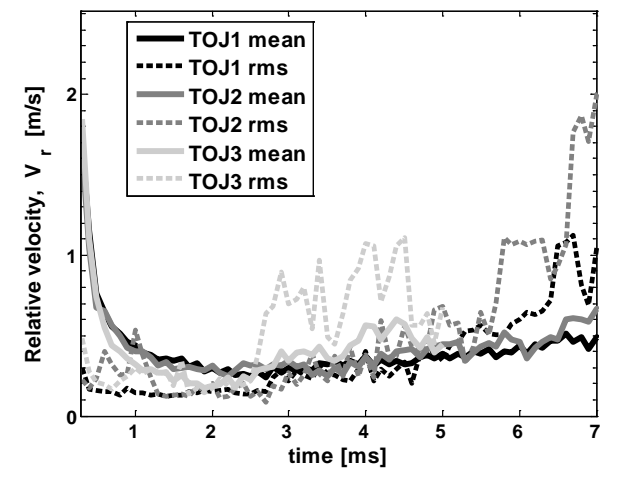

Fig. 5: Mean and r.m.s. of the speed of the edge flames relative to the flow against time from the spark initiation. Spark at $r=0, z=0$.

Single-column width 


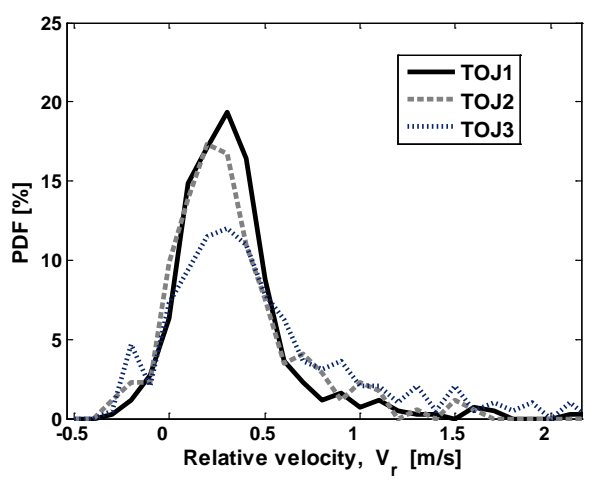

(a)

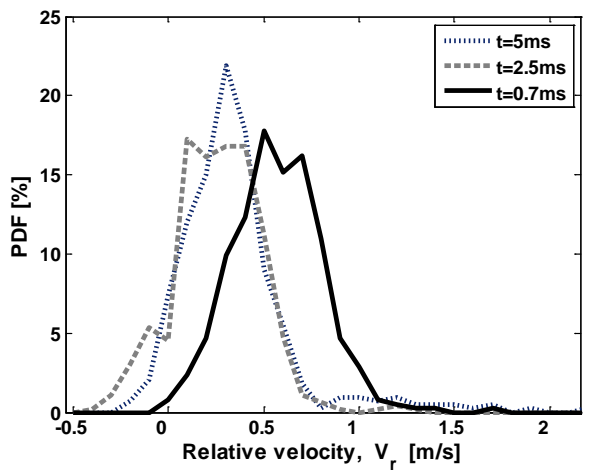

(b)

Fig. 6: Probability density function (PDF) of the relative propagation velocity of (a) the three flames at 4 ms after the spark, and (b) flame TOJ1 at different time delays from the spark.

Single-column width 


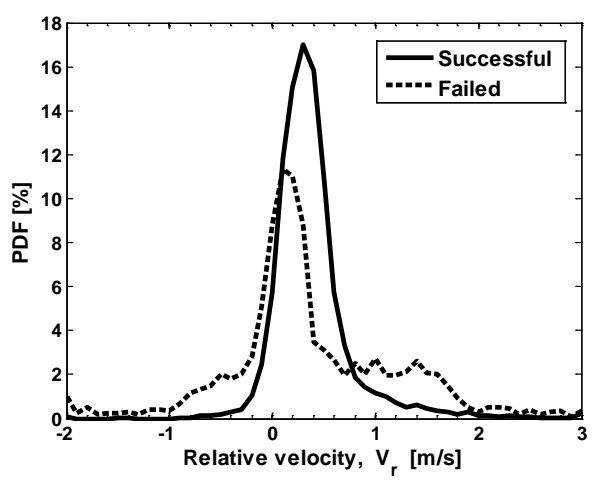

Fig. 7: Comparison between the pdf of the relative velocity for the successful and failed ignition events for flame TOJ1. Spark at $r=0, z=0$

Single-column width 